\title{
The large-scale blast score ratio (LS-BSR) pipeline: a method to rapidly compare genetic content between bacterial genomes
}

Background. As whole genome sequence data from bacterial isolates becomes cheaper to generate, computational methods are needed to correlate sequence data with biological observations. Here we present the large-scale BLAST score ratio (LS-BSR) pipeline, which rapidly compares the genetic content of hundreds to thousands of bacterial genomes, and returns a matrix that describes the relatedness of all coding sequences (CDSs) in all genomes surveyed. This matrix can be easily parsed in order to identify genetic relationships between bacterial genomes. Although pipelines have been published that group peptides by sequence similarity, no other software performs the rapid, large-scale, full-genome comparative analyses carried out by LS-BSR. Results. To demonstrate the utility of the method, the LS-BSR pipeline was tested on 96 Escherichia coli and Shigella genomes; the pipeline ran in 163 minutes using 16 processors, which is a greater than 7-fold speedup compared to using a single processor. The BSR values for each CDS, which indicate a relative level of relatedness, were then mapped to each genome on an independent core genome single nucleotide polymorphism (SNP) based phylogeny. Comparisons were then used to identify clade specific CDS markers and validate the LS-BSR pipeline based on molecular markers that delineate between classical E. coli pathogenic variant (pathovar) designations. Scalability tests demonstrated that the LS-BSR pipeline can process 1,000 E. coli genomes in $27-57 \mathrm{~h}$, depending upon the alignment method, using 16 processors.

Conclusions. LS-BSR is an open-source, parallel implementation of the BSR algorithm, enabling rapid comparison of the genetic content of large numbers of genomes. The results of the pipeline can be used to identify specific markers between user-defined phylogenetic groups, and to identify the loss and/or acquisition of genetic information between bacterial isolates. Taxa-specific genetic markers can then be translated into clinical diagnostics, or can be used to identify broadly conserved putative therapeutic candidates. 
1 Authors:

2 Jason W. Sahl*

3 Division of Pathogen Genomics

4 Translational Genomics Research Institute

53051 W. Shamrell Blvd, Suite 106

6 Flagstaff, AZ, 86001, USA

7 Email: jsahl@tgen.org

8 J. Gregory Caporaso

9 Department of Biological Sciences

10 Northern Arizona University

111298 S. Knoles Drive, PO Box 4073

12 Flagstaff, AZ, 86011, USA

13 Email: gregcaporaso@gmail.com

14 David A. Rasko

15 Department of Microbiology and Immunology

16 University of Maryland School of Medicine

17 Baltimore, MD, 21202, USA

18 Email: drasko@som.umaryland.edu

19 Paul Keim

20 Center for Microbial Genetics and Genomics

21 Northern Arizona University

221298 S. Knoles Drive, PO Box 4073

23 Flagstaff, AZ, 86011, USA

24 Email: paul.keim@nau.edu

$25{ }^{*}$ To whom correspondence should be addressed 
Whole genome sequence (WGS) data has changed our view of bacterial relatedness and evolution. Computational analyses available for WGS data include, but are not limited to, single nucleotide polymorphism (SNP) discovery (DePristo et al. 2011), core genome phylogenetics (Sahl et al. 2011), and gene based comparative methods (Hazen et al. 2013; Sahl et al. 2013). In 2005, a BLAST score ratio (BSR) method was introduced in order to compare peptide identity from a limited number of bacterial genomes (Rasko et al. 2005). However, the "all vs. all" implementation of this method scales poorly with a larger number of sequenced genomes. gene content of a large number of bacterial genomes. Comparable methods have been published in order to group genes into gene families, including OrthoMCL ( $\underline{\text { Li et al. }}$ 2003), TribeMCL (Enright et al. 2002), and GETHOGs (Altenhoff et al. 2013). Although grouping peptides into gene families is not the primary focus of LS-BSR, the output can be parsed to identify the pan-genome (Tettelin et al. 2008) structure of a species; scripts are included with LS-BSR that classify coding sequences (CDSs) into pan-genome categories based on user-defined identity thresholds.

Pipelines have also been established to perform comprehensive pan-genome analyses, including the pan-genome analysis pipeline (PGAP) (Zhao et al. 2012), which requires specific gene annotation from GenBank and complicates the analysis of large numbers of novel genomes. PGAP also doesn't allow for the screen of specific genes of interest against query genomes in order to identify patterns of distribution. GET_HOMOLOGUES (Contreras-Moreira \& Vinuesa 2013) is a recently published tool that can be used for pan-genome analyses, including the generation of dendrograms based on the presence/absence of homologous genes; by only using presence/absence based on gene homology, more distantly related gene relatedness cannot be fully investigated. The integrated toolkit for the exploration of microbial pan-genomes (ITEP) toolkit (Benedict et al. 2014) was recently published and performs similar functions to LS-BSR, including the identification of gene gain/loss at nodes of a phylogeny. ITEP relies on multiple dependencies and workflows, which are available as a pre-packaged virtual machine. The authors of ITEP report that an analysis of 200 diverse genomes would take $\sim 6$ days on a server with 12 processors and scales quadratically with additional genomes. 
The LS-BSR method can either use a defined set of genes, or can use Prodigal (Hyatt et al. 2010) to predict CDSs from a set of query genomes. When using Prodigal, all CDSs are concatenated and then de-replicated using USEARCH (Edgar 2010) at a pairwise identity of 0.9 (identity threshold can be modified by the user). Each unique CDS is then translated with BioPython (www.biopython.org) and aligned against its nucleotide sequence with TBLASTN (Altschul et al. 1997) to calculate the reference bit score; if BLASTN or BLAT (Kent 2002) is invoked, the nucleotide sequences are aligned. Each query sequence is then aligned against each genome with BLAT, BLASTN, or TBLASTN and the query bit score is tabulated. The BSR value is calculated by dividing the query bit score by the reference bit score, resulting in a BSR value between 0.0 and 1.0 (values slightly higher than 1.0 have been observed due to variable bit score values obtained by TBLASTN). The results of the LS-BSR pipeline include a matrix that contains each unique CDS name and the BSR value in each genome surveyed. CDSs that have more than one significant BSR value in at least one genome are also identified in the output. A separate file is generated for CDSs where one duplicate is significantly including RAST (Aziz et al. 2008) and prokka

86 (http://www.vicbioinformatics.com/software.prokka.shtml). LS-BSR source code, unit 87 tests, and test data can be freely obtained at https://github.com/jasonsahl/LS-BSR under a GNU GPL v3 license.

\section{RESULTS AND DISCUSSION}


90 LS-BSR algorithm speed and scalability. To determine the scalability of the LS-BSR

91 method, 1,000 Escherichia coli and Shigella genomes were downloaded from Genbank

92 (Benson et al. 2012); E. coli was used as a test case due to the large number of

93 genomes deposited in Genbank. Genomes were sub-sampled at different depths (100

94 through 1000, sampling every 100) with a python script

95 (https://gist.github.com/jasonsahl/115d22bfa35ac932d452) and processed with LS-BSR

96 using 16 processors. A plot of wall time and the number of genomes processed

97 demonstrates the scalability of the method (Figure 1A) using three different alignment

98 methods. To demonstrate the parallel nature of the algorithm, $100 \mathrm{E}$. coli genomes were

99 processed with different numbers of processors. The results demonstrate decreased

100 runtime of LS-BSR with an increase in the number of processors used (Figure 1B).

101 Improvements on a previous BSR implementation. The LS-BSR method is an 102 improvement on a previous BSR implementation (http://bsr.igs.umaryland.edu/) in terms 103 of speed and ease of use. The former BSR algorithm (Rasko, et al., 2005) requires 104 peptide sequences and genomic coordinates of CDSs to run. LS-BSR only requires 105 genome assemblies in FASTA format, which is the standard output of most genome 106 assemblers. To test the speed differences between methods, $10 \mathrm{E}$. coli genomes 107 (Supplemental Table 1) were processed with both methods. Using the same number of 108 processors $(n=2)$ on the same server, the original BSR method took $\sim 14$ hours (wall 109 time) to complete, while the LS-BSR method, using TBLASTN, took $\sim 25$ minutes to 110 complete (wall time). Because the original BSR method is an "all vs. all" comparison 111 and the LS-BSR method is a "one vs. all" comparison, this difference is expected to be 112 more pronounced as the number of genomes analyzed increases.

113 Test case: analysis of $96 \mathrm{E}$. coli and Shigella genomes. To demonstrate the utility of 114 the LS-BSR pipeline, a set of 96 E. coli and Shigella genomes were processed 115 (Supplemental Table 1); these genomes are in various stages of assembly 116 completeness and have been generated with various sequencing technologies from 117 Sanger to Illumina. The BSR matrix was generated with TBLASTN in $2 \mathrm{~h} 34 \mathrm{~m}$ from a set 118 of $\sim 20,000$ unique CDSs using 16 processors. In addition to the LS-BSR analysis, a 119 core genome single nucleotide polymorphism (SNP) phylogeny was inferred on 96 120 genomes using methods published previously (Sahl et al. 2011); the SNP phylogeny 
121 with labels is shown in Supplemental Figure 1. Briefly, all genomes were aligned with

122 Mugsy (Angiuoli \& Salzberg 2010) and the core genome was extracted from the whole 123 genome alignment; the alignment file was then converted into a multiple sequence 124 alignment in FASTA format. Gaps in the alignment were removed with Mothur (Schloss 125 et al. 2009) and a phylogeny was inferred on the reduced alignment with FastTree2 126 (Price et al. 2010).

The compare_BSR.py script included with LS-BSR was used to identity CDS 128 markers that are unique to specific phylogenetic clades (Figure 2). Identified CDSs had 129 a BSR value $\geq 0.8$ in targeted genomes and a BSR value $<0.4$ in non-targeted genomes; 130 the gene annotation of all marker CDSs is detailed in Supplemental Table 2. The 131 conservation and distribution of all clade-specific markers was visualized by correlating 132 the phylogeny with a heatmap of BSR values (Figure 2). This presentation provides an 133 easy way for the user to highlight features conserved in one or more phylogenomic 134 clades.

135 E. coli and Shigella pathogenic variants (pathovars) are delineated by the presence 136 of genetic markers primarily present on mobile genetic elements (Rasko et al. 2008). 137 The conservation of these markers was used as a validation of the LS-BSR method. A 138 representative sequence from each pathovar-specific marker (Supplemental Table 2) 139 was screened against the 96-genome test set and the BSR values (Supplemental Table 140 3) were visualized as a heatmap (Figure 2). The BSR matrix demonstrates that 141 pathovar-specific genes were accurately identified in each targeted genome 142 (Supplemental Table 3, Figure 2). For example, the ipaH3 marker was positively 143 identified in all Shigella genomes and the Shiga toxin gene (stx2a) was conserved in the 144 clade including 0157:H7 E. coli (Figure 2). A sub-set of these $96 \mathrm{E}$. coli genomes is 145 included with LS-BSR as test data to characterize the conservation and distribution of 146 pathovar specific genes.

147 Finally, the BSR values were used to cluster all 96 genomes with an average linkage 148 algorithm implemented in $\mathrm{MeV}$ and the structure of the resulting dendrogram was 149 compared to the core SNP phylogeny. The BSR based clustering method incorporates 150 both the core and accessory genome, while the SNP phylogeny relies on core genomic 151 regions alone. A comparison of the tree structures demonstrates that while Shigella 152 genomes share a diverse evolutionary history (Figure $3 \mathrm{~A}$ ), they all cluster together 153 based on gene presence and conservation (Figure 3B). This result was also observed 
154 using a k-mer frequency method (Sims \& Kim 2011), which uses all possible k-mer

155 values to infer a phylogeny and validates the findings of the LS-BSR pipeline. The

156 dendrogram also differed from the core SNP phylogeny in other genomes, which could

157 represent either assembly problems, or more likely the acquisition of accessory genomic

158 regions that are not a product of direct descent.

159 The functionality of LS-BSR was compared to recently released pan-genome 160 software packages including GET_HOMOLOGUES (Contreras-Moreira \& Vinuesa 161 2013), ITEP (Benedict et al. 2014), and PGAP (Zhao et al. 2012). A set of 11 162 Streptococcus pyogenes genomes was chosen for the comparative analysis, as it was 163 also used as a test set in the PGAP publication; the comparative analysis and results 164 are shown in Table 1. Overall, the size of the core genome was comparable between 165 methods, with LS-BSR (BLASTN) and GET-HOMOLOGUS calculating differing core 166 genome numbers compared to the other methods. However, small differences were 167 expected due to differing thresholds and clustering algorithms. Based on these results, 168 LS-BSR represent a significant improvement in terms of speed and ease of use 169 compared to comparable methods, while having comparable utility.

170 Pan-genome analyses. One application in comparative genomics is the analysis of the 171 pan-genome, or the combined genome, of isolates within a species. Post matrix172 building scripts are available to visualize the pan-genome of a given dataset. One script 173 (BSR_to_PANGP.py) creates a matrix compatible with PanGP (Zhao et al. 2014), for 174 visualization of pan-genome statistics. The pan_genome_stats.py script provides data 175 that can be used to visualize the conservation of CDSs at different genome depths 176 (Figure 4A). An additional script randomly subsamples the CDS distribution at all depths 177 and produces data that can be plotted to visualize core genome convergence (Figure 178 4B), accumulation of CDSs (Figure 4C), and the number of unique CDSs for each 179 genome analyzed (Figure 4D). All analyses were conducted on a set of $100 \mathrm{E}$. coli 180 genomes, with 100 iterations.

\section{CONCLUSIONS}

182 The LS-BSR method can rapidly compare the gene content of a relatively large 183 number of bacterial genomes in either draft or complete form, though with more 184 fragmented assemblies LS-BSR is likely to perform sub-optimally. As sequence read 
lengths improve, assembly fragmentation should become less problematic due to more contiguous assemblies. LS-BSR can also be used to rapidly screen a collection of genomes for the conservation of known virulence factors or genetic features. By using a range of peptide relatedness, instead of a defined threshold, homologs and paralogs can also be identified for further characterization.

LS-BSR is written in python, with many steps conducted in parallel. This allows the script to scale well from hundreds to thousands of genomes. The LS-BSR method is a major improvement on a previous BSR implementation in terms of speed, ease of use, and utility. As more WGS data from bacterial genomes become available, methods will be required to quickly compare their genetic content and perform pan-genome analyses. LS-BSR is an open-source software package to rapidly perform these comparative genomic workflows.

\section{ACKNOWLEDGEMENTS}

Thanks to Darrin Lemmer for his critical review of the LS-BSR code.

\section{REFERENCES}

Altenhoff AM, Gil M, Gonnet GH, and Dessimoz C. 2013. Inferring hierarchical orthologous groups from orthologous gene pairs. PLoS ONE 8:e53786.

Altschul SF, Madden TL, Schaffer AA, Zhang J, Zhang Z, Miller W, and Lipman DJ. 1997. Gapped BLAST and PSI-BLAST: a new generation of protein database search programs. Nucleic Acids Res 25:3389-3402.

Angiuoli SV, and Salzberg SL. 2010. Mugsy: Fast multiple alignment of closely related whole genomes. Bioinformatics.

Aziz RK, Bartels D, Best AA, DeJongh M, Disz T, Edwards RA, Formsma K, Gerdes S, Glass EM, Kubal M, Meyer F, Olsen GJ, Olson R, Osterman AL, Overbeek RA, McNeil LK, Paarmann D, Paczian T, Parrello B, Pusch GD, Reich C, Stevens R, Vassieva O, Vonstein V, Wilke A, and Zagnitko O. 2008. The RAST Server: rapid annotations using subsystems technology. BMC genomics 9:75.

Benedict MN, Henriksen JR, Metcalf WW, Whitaker RJ, and Price ND. 2014. ITEP: An integrated toolkit for exploration of microbial pan-genomes. BMC genomics 15:8.

Benson DA, Karsch-Mizrachi I, Clark K, Lipman DJ, Ostell J, and Sayers EW. 2012. GenBank. Nucleic Acids Res 40:D48-53.

Bork P, Hugenholtz P, Kunin V, Raes J, Harris JK, Spear JR, Walker JJ, Ivanova N, von Mering C, Bebout BM, and Pace NR. 2008. Millimeter-scale genetic gradients and community-level molecular convergence in a hypersaline microbial mat. $\mathrm{Mol}$ Sys Biol 4:198-198. 
Contreras-Moreira B, and Vinuesa P. 2013. GET HOMOLOGUES, a versatile software package for scalable and robust microbial pangenome analysis. Appl Environ Microbiol 79:7696-7701.

DePristo MA, Banks E, Poplin R, Garimella KV, Maguire JR, Hartl C, Philippakis AA, del Angel G, Rivas MA, Hanna M, McKenna A, Fennell TJ, Kernytsky AM, Sivachenko AY, Cibulskis K, Gabriel SB, Altshuler D, and Daly MJ. 2011. A framework for variation discovery and genotyping using next-generation DNA sequencing data. Nature genetics 43:491-498.

Edgar RC. 2010. Search and clustering orders of magnitude faster than BLAST. Bioinformatics 26:2460-2461.

Enright AJ, Van Dongen S, and Ouzounis CA. 2002. An efficient algorithm for large-scale detection of protein families. Nucleic acids research 30:1575-1584.

Hazen TH, Sahl JW, Fraser CM, Donnenberg MS, Scheutz F, and Rasko DA. 2013. Refining the pathovar paradigm via phylogenomics of the attaching and effacing Escherichia coli. Proc Natl Acad Sci U S A 110:12810-12815.

Hyatt D, Chen GL, Locascio PF, Land ML, Larimer FW, and Hauser LJ. 2010. Prodigal: prokaryotic gene recognition and translation initiation site identification. $B M C$ Bioinformatics 11:119.

Kent WJ. 2002. BLAT--the BLAST-like alignment tool. Genome Res 12:656-664.

Li L, Stoeckert CJ, Jr., and Roos DS. 2003. OrthoMCL: identification of ortholog groups for eukaryotic genomes. Genome Res 13:2178-2189.

Price MN, Dehal PS, and Arkin AP. 2010. FastTree 2--approximately maximumlikelihood trees for large alignments. PLoS ONE 5:e9490.

R Core Team RCT. 2013. R: A language and environment for statistical computing. Available at http://www.R-project.org.

Rasko DA, Myers GS, and Ravel J. 2005. Visualization of comparative genomic analyses by BLAST score ratio. BMC Bioinformatics 6:2.

Rasko DA, Rosovitz MJ, Myers GS, Mongodin EF, Fricke WF, Gajer P, Crabtree J, Sebaihia M, Thomson NR, Chaudhuri R, Henderson IR, Sperandio V, and Ravel J. 2008. The pangenome structure of Escherichia coli: comparative genomic analysis of $E$. coli commensal and pathogenic isolates. J Bacteriol 190:68816893.

Saeed Al, Bhagabati NK, Braisted JC, Liang W, Sharov V, Howe EA, Li J, Thiagarajan M, White JA, and Quackenbush J. 2006. TM4 microarray software suite. Methods Enzymol 411:134-193.

Sahl JW, Gillece JD, Schupp JM, Waddell VG, Driebe EM, Engelthaler DM, and Keim P. 2013. Evolution of a pathogen: a comparative genomics analysis identifies a genetic pathway to pathogenesis in Acinetobacter. PLoS ONE 8:e54287.

Sahl JW, Steinsland H, Redman JC, Angiuoli SV, Nataro JP, Sommerfelt H, and Rasko DA. 2011. A comparative genomic analysis of diverse clonal types of enterotoxigenic Escherichia coli reveals pathovar-specific conservation. Infect Immun 79:950-960.

Schloss PD, Westcott SL, Ryabin T, Hall JR, Hartmann M, Hollister EB, Lesniewski RA, Oakley BB, Parks DH, Robinson CJ, Sahl JW, Stres B, Thallinger GG, Van Horn DJ, and Weber CF. 2009. Introducing mothur: Open-Source, PlatformIndependent, Community-Supported Software for Describing and Comparing Microbial Communities. Appl Environ Microbiol 75:7537-7541. 
Sims GE, and Kim SH. 2011. Whole-genome phylogeny of Escherichia coli/Shigella group by feature frequency profiles (FFPs). Proc Natl Acad Sci U S A 108:83298334.

Tettelin H, Riley D, Cattuto C, and Medini D. 2008. Comparative genomics: the bacterial pan-genome. Curr Opin Microbiol 11:472-477.

Zhao Y, Jia X, Yang J, Ling Y, Zhang Z, Yu J, Wu J, and Xiao J. 2014. PanGP: A tool for quickly analyzing bacterial pan-genome profile. Bioinformatics.

Zhao Y, Wu J, Yang J, Sun S, Xiao J, and Yu J. 2012. PGAP: pan-genomes analysis pipeline. Bioinformatics 28:416-418.

\section{Figure Legends:}

Figure 1. Time performance of the LS-BSR pipeline. Panel A) 1000 Escherichia coli and Shigella genomes were randomly sub-sampled and analyzed using default LS-BSR parameters and 16 processors. Wall time was plotted against the number of genomes analyzed. The results demonstrate that the LS-BSR pipeline scales well with increasing numbers of genomes. Panel B) The same set of 100 E. coli genomes was processed with different numbers of processors and the wall time was plotted. The results demonstrate that using additional processors decreases the overall run time of LS-BSR.

Figure 2. The distribution of virulence factors and phylogenomic markers associated with a core single nucleotide polymorphism (SNP) phylogeny. The core SNP phylogeny was inferred from a whole genome alignment produced by Mugsy (Angiuoli \& Salzberg 2010). Known virulence genes (Supplemental Table 2) were screened against 96 Escherichia coli and Shigella genomes using BLASTN within LS-BSR. Clade specific markers were identified at defined nodes in the phylogeny (A through Q). Gene annotations for these markers are detailed in Supplemental Table 2.

Figure 3. A comparison of 96 Escherichia coli / Shigella genomes between (Panel A) a core single nucleotide polymorphism (SNP) phylogeny or (Panel B) a cluster generated with the Multiple Experiment Viewer (Saeed et al. 2006) from BLAST Score Ratio (BSR) values that include the entire pan-genome. Colors applied to each classical $E$. coli phylogroup were applied to the SNP phylogeny and transferred to the BSR cladogram. Shigella genomes are marked with a red circle. 
297 Figure 4. Pan-genome plots generated from LS-BSR output. Analyses were conducted 298 on a set of 100 Escherichia coli genomes. The distribution of coding region sequences 299 (CDSs) across the set of genomes surveyed is shown in Panel A. A supplemental script 300 can be used to better understand the convergence of the core genome (Panel B), the 301 accumulation of CDSs (Panel C), and the number of unique CDSs for each genome 302 analyzed (Panel D); each analysis was conducted with 100 random sum-samplings and 303 means are depicted with red diamonds.

304 Supplemental Figure 1. A core genome SNP phylogeny of 96 Escherichia coli and 305 Shigella genomes. The core genome was extracted from the output of Mugsy (Angiuoli 306 \& Salzberg 2010) and the phylogeny was inferred with FastTree2 (Price et al. 2010). 307 This phylogeny contains labels that can be used to identify specific genomes in Figures 3082 and 3. 


\section{Table 1 (on next page)}

Comparison of four pan-genome methods on a test set of 11 Streptococcus pyogenes genomes 
Table 1. Comparison of four pan-genome methods on a test set of 11 Streptococcus pyogenes genomes

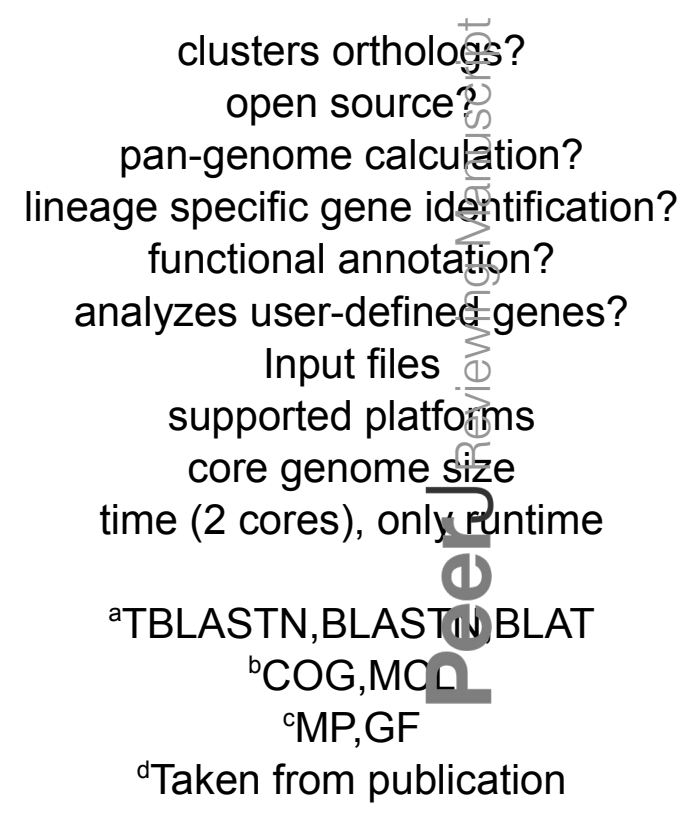

${ }^{\mathrm{d}}$ Taken from publication

\begin{tabular}{|c|c|c|c|}
\hline LS-BSR & GET_HOMOLOGUES & PGAP & ITEP \\
\hline Yes & Yes & Yes & Yes \\
\hline Yes & Yes & Yes & Yes \\
\hline Yes & Yes & Yes & Yes \\
\hline Yes & Yes & Yes & Yes \\
\hline No & Yes & Yes & Yes \\
\hline Yes & No & No & Yes \\
\hline ".fasta" & GenBank or ".faa" & ".faa",".fna",".ppt" & GenBank \\
\hline linux, OSX & linux/OSX & linux & linux/OS $x$ \\
\hline $1318,1350,1426^{a}$ & $1232,1234^{b}$ & $1332,1366^{c, d}$ & 1342 \\
\hline $5 \mathrm{~m} 59 \mathrm{~s}, 1 \mathrm{~m} 53 \mathrm{~s}, 1 \mathrm{~m} 17 \mathrm{~s}^{\mathrm{a}}$ & $25 \mathrm{~m} 14 \mathrm{~s}$ & $29 m 59 s, 199 m 58 s^{c, d}$ & $24 \mathrm{~m} 22 \mathrm{~s}$ \\
\hline
\end{tabular}




\section{Figure 1}

Time performance of the LS-BSR pipeline.

Panel A) 1000 Escherichia coli and Shigella genomes were randomly sub-sampled and analyzed using default LS-BSR parameters and 16 processors. Wall time was plotted against the number of genomes analyzed. The results demonstrate that the LS-BSR pipeline scales well with increasing numbers of genomes. Panel B) The same set of 100 E. coli genomes was processed with different numbers of processors and the wall time was plotted. The results demonstrate that using additional processors decreases the overall run time of LSBSR. 


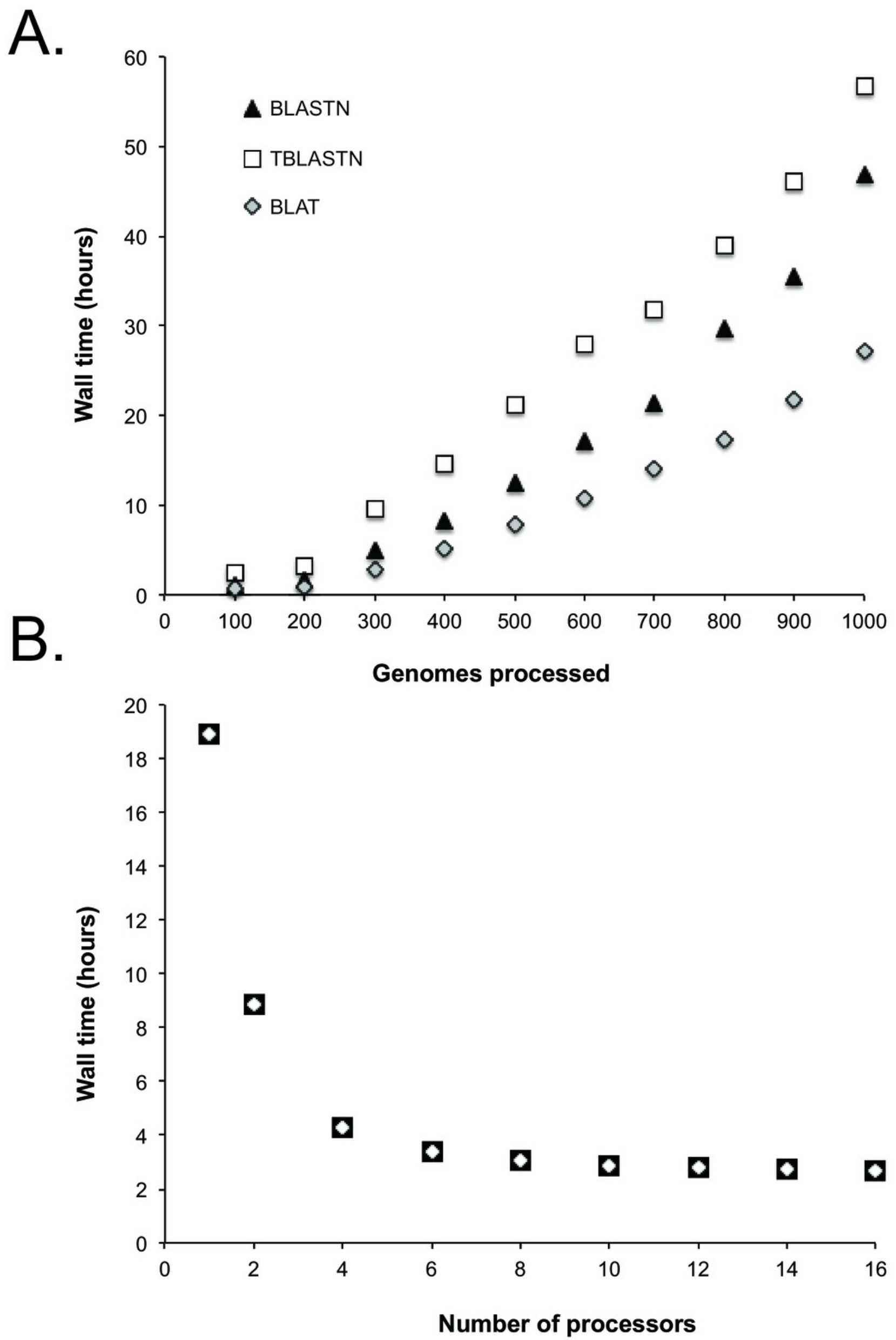




\section{Figure 2}

The distribution of virulence factors and phylogenomic markers associated with a core single nucleotide polymorphism (SNP) phylogeny

The core SNP phylogeny was inferred from a whole genome alignment produced by Mugsy ( Angiuoli \& Salzberg 2010 ). Known virulence genes (Supplemental Table 2) were screened against 96 Escherichia coli and Shigella genomes using BLASTN within LS-BSR. Clade specific markers were identified at defined nodes in the phylogeny (A through $Q$ ). Gene annotations for these markers are detailed in Supplemental Table 2. 


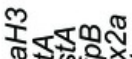

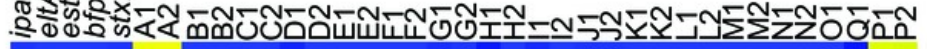

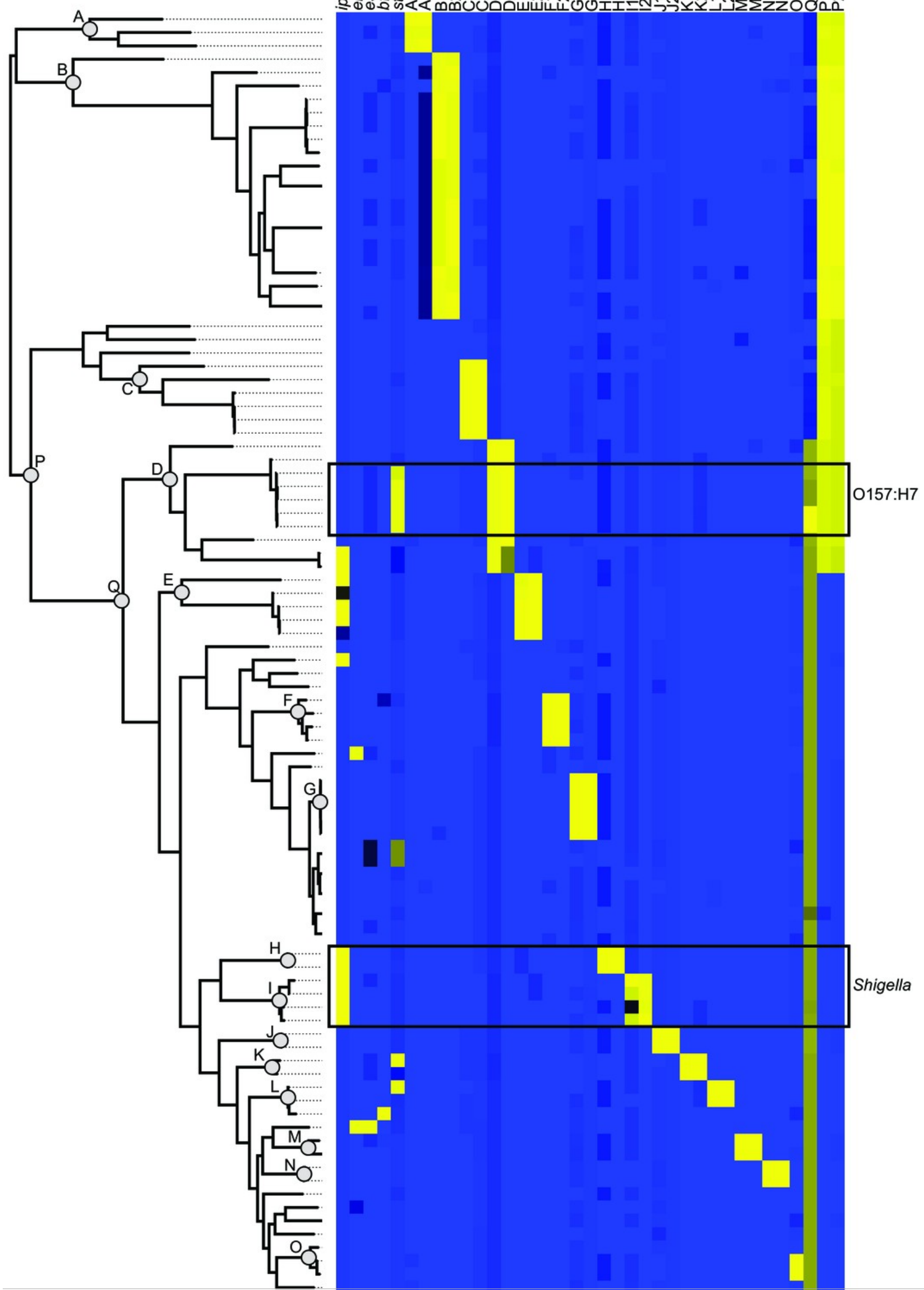




\section{Figure 3}

Comparison of LS-BSR cluster with core genome SNP phylogeny

A comparison of 96 Escherichia coli / Shigella genomes between (Panel A) a core single nucleotide polymorphism (SNP) phylogeny or (Panel B) a cluster generated with the Multiple Experiment Viewer ( Saeed et al. 2006 ) from BLAST Score Ratio (BSR) values that include the entire pan-genome. Colors applied to each classical E. coli phylogroup were applied to the SNP phylogeny and transferred to the BSR cladogram. Shigella genomes are marked with a red circle. 
A.

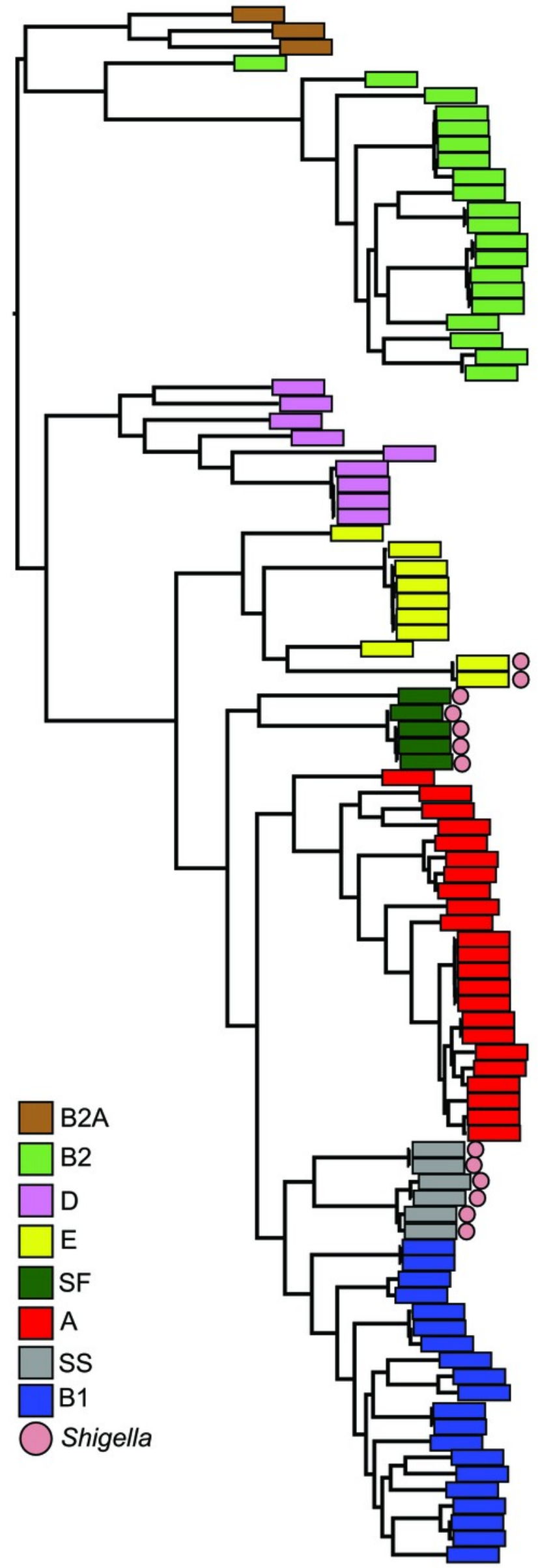

B.

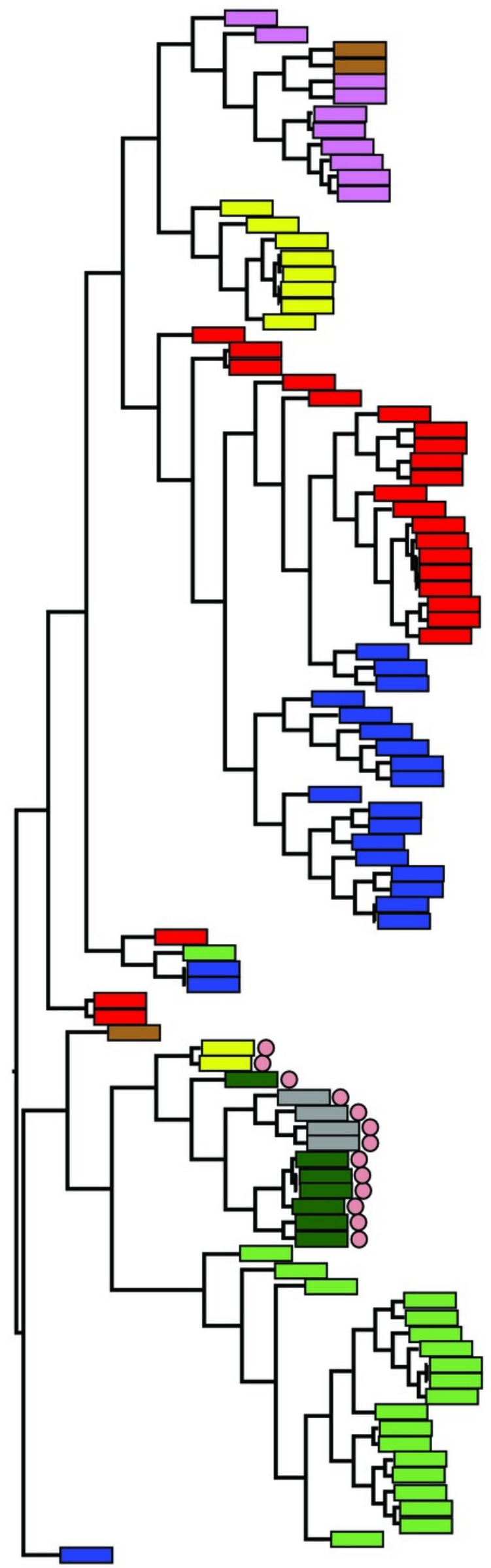




\section{Figure 4}

\section{Pan-genome plots generated from LS-BSR output}

Analyses were conducted on a set of 100 Escherichia coli genomes. The distribution of coding region sequences (CDSs) across the set of genomes surveyed is shown in Panel A. A supplemental script can be used to better understand the convergence of the core genome (Panel B), the accumulation of CDSs (Panel C), and the number of unique CDSs for each genome analyzed (Panel D); each analysis was conducted with 100 random sum-samplings and means are depicted with red diamonds.

A.

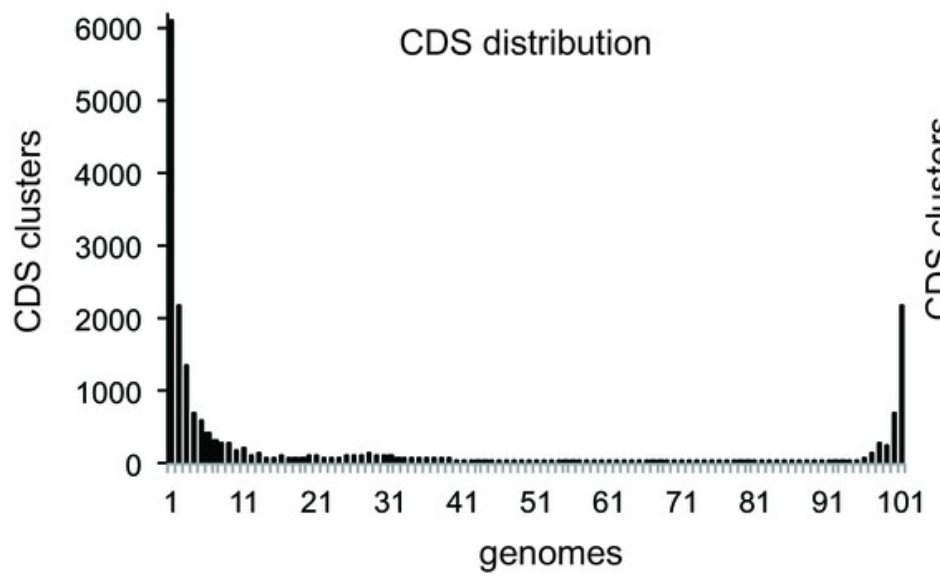

B.

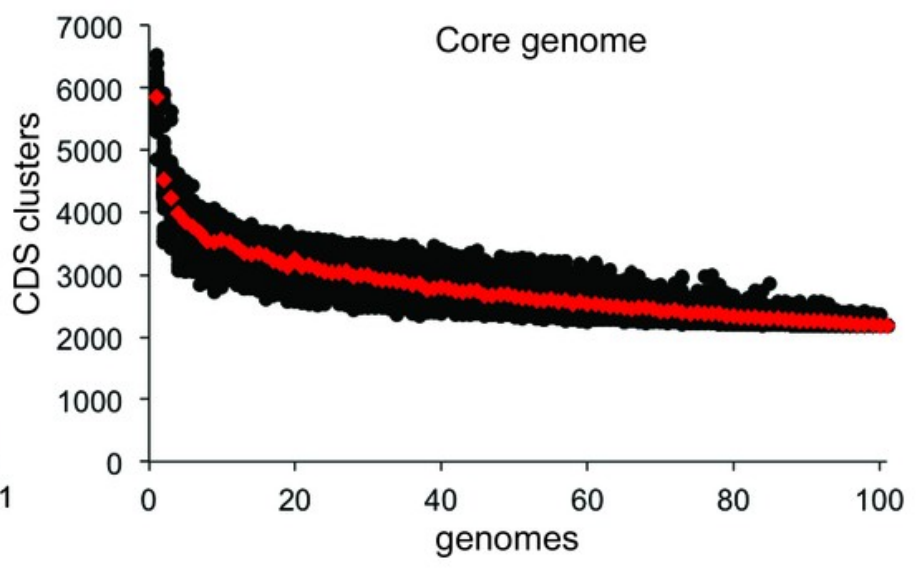

C.

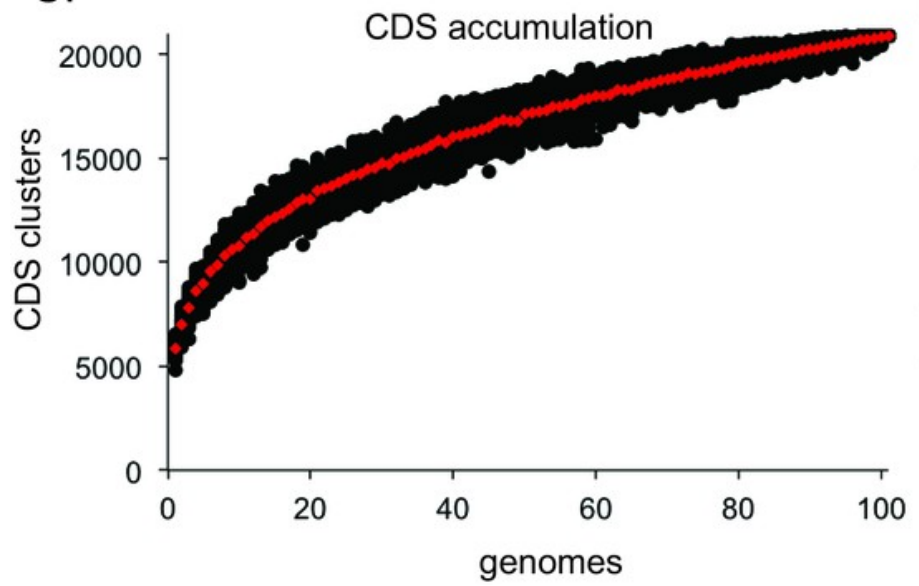

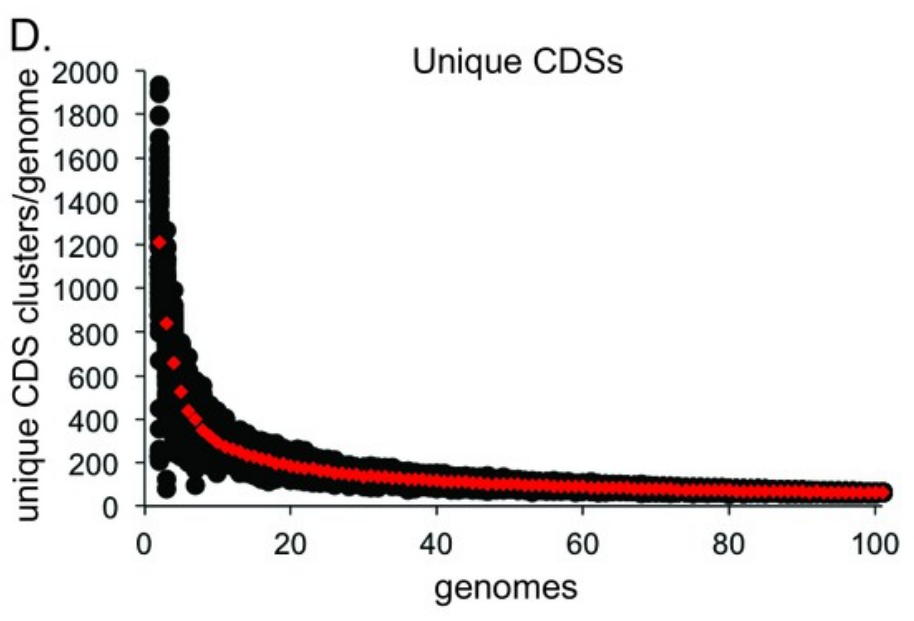

